\title{
Redescubrimiento de una especie y su nueva clasificación como subespecie de Alstroemeria garaventae (Alstroemeriaceae) de Chile
}

\author{
Rediscovery of a species and its new classification as a subspecies of Alstroemeria garaventae \\ (Alstroemeriaceae) de Chile
}

\author{
Mélica Muñoz-Schick ${ }^{1 *}$, María Teresa Eyzaguirre ${ }^{2}$ \& Andrés Moreira-Muñoz ${ }^{3}$ \\ ${ }^{1}$ Museo Nacional de Historia Natural, interior Quinta Normal, Casilla 787, Santiago, Chile. \\ ${ }^{2}$ Fundación R.A. Philippi de Estudios Naturales, Suecia 1323, Depto. 403, Santiago. \\ ${ }^{3}$ Instituto de Geografía, Pontificia Universidad Católica de Valparaíso, Avenida Brasil 2241, Valparaíso. \\ *Email: melica4@gmail.com
}

\begin{abstract}
We redescribed an uncertain species from the Achibueno and Ancoa valleys, Linares province, Chile. We have changed its status to a subspecies: Alstroemeria garaventae subsp. longaviensis. It differs from the typical subspecies in the minor size of the tepals and of the inflorescense leaves, shows a southern distribution and grows at lower altitude than the typical subspecies.
\end{abstract}

El género Alstroemeria es estrictamente sudamericano, abarcando desde Venezuela $\left(3^{\circ} \mathrm{N}\right)$ hasta Tierra del Fuego $\left(53^{\circ}\right.$ S). La distribución muestra una marcada disyunción entre los andes australes (Chile-Argentina) y el SE de Brasil.

Según Muñoz-Schick y Moreira-Muñoz (2003), la mayor cantidad de taxones se encuentra en Chile (49), luego en Brasil (39), Argentina (10), Perú (7) y Bolivia, Uruguay, Paraguay y Venezuela con una sola especie. Recientemente ha aparecido una nueva publicación sobre este género (Finot et al. 2018), en la que se indican 58 taxones aceptados para Chile.

La variabilidad morfológica floral en el género en Chile es muy grande, las especies presentan flores de colorido y dibujos variables en una misma especie. Ruiz et al. (2010) han indicado esta variabilidad en la especie A. hookeri Lodd. subsp. hookeri y Baeza et al. (2016) en A. diluta Ehr. Bayer.

Los tépalos internos superiores son generalmente los que poseen mayor variabilidad en el colorido, sin embargo, hay especies que presentan dibujos (rayas o puntos) en todos los tépalos. Este caso se ha registrado para las especies A. garaventae Ehr. Bayer (flores rosadas), A. versicolor Ruiz et Pav. (flores rosado-amarillentas) y solo en los ápices de los externos (siempre los internos con rayas) en $A$. achirae Muñoz-Schick et Brinck (flores amarillas) y en A. spathulata
C. Presl (flores rosadas). También se ha observado esta ornamentación en todos los tépalos, en poblaciones andinas y de la cordillera de la Costa de A. pallida Graham (García 2010), la cual generalmente presenta dibujos sólo en los tépalos internos superiores.

Las especies de flores pequeñas nortinas como $A$. andina Phil. y A. polyphylla Phil. también presentan rayas en casi todos los tépalos y adicionalmente se ha observado este patrón en poblaciones de A. angustifolia Herb., del sur de la Región de Coquimbo.

En un recorrido por localidades aledañas al río Achibueno, Ancoa y Loncomilla, nos encontramos en diferentes años con una especie de Alstroemeria que nos llamó la atención por sus tépalos con rayas en todos ellos. Al analizar la bibliografía específica nos encontramos con una especie descrita para la zona, por Ravenna en 1988, que él llamó A. longaviensis.

En la imposibilidad de consultar el ejemplar Tipo ya que no está depositado en los herbarios indicados por el autor (NY, SGO), y dado que hay un ejemplar en SGO recolectado posteriormente en la localidad La Balsa, indicada por dicho autor, hemos concluido que la nuestra corresponde al mismo taxón de Ravenna.

Al analizar todas estas especies similares, indicadas más 
arriba, hemos concluido que, por la forma y ornamentación constante de los tépalos, es semejante a A. garaventae (Bayer 1987) y sin diferencias morfológicas tan notables como para designar una especie nueva, corresponde designarla como subespecie de aquella.

Alstroemeria garaventae Ehr. Bayer subsp. longaviensis (Ravenna) Muñoz-Schick \& Eyzag. subsp. nov.

NEOTIPO: Región del Maule, provincia de Linares, camino desde Embalse Ancoa hacia poblado de Ancoa, camping Los

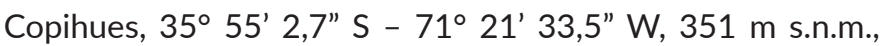
13-I-2016, Muñoz-Schick 5630 (Neotipo SGO, Isoneotipo CONC).

Basiónimo: Alstroemeria longaviensis Ravenna Phytologia 64(4): 285, 1988. Linares, valle río Longaví, nov. 1971, La Balsa, Ravenna 1630, Herb. Ravenna (Holotipo) NY, SGO isotipos [?] no depositados.

Hierba con tallos erectos, débiles, de $40-65 \mathrm{~cm}$ de largo; rizoma cilíndrico de $8,5 \mathrm{~cm}$ de largo y 4-6 $\mathrm{mm}$ de diámetro, enterrado a 12 - $14 \mathrm{~cm}$ de profundidad; raíces filiformes de 1 $\mathrm{mm}$ de diámetro, abundantes, una parte de ellas engrosadas a los 4 a $8 \mathrm{~cm}$, formando tubérculos fusiformes, irregulares, de 3 a $8 \mathrm{~cm}$ de largo por 1-1,2 cm de diámetro y terminados en una corta raíz delgada (Fig. 1).

Hojas lanceolado-lineares, de $1,5-7 \mathrm{~cm}$ de largo por 2 $\mathrm{mm}$ de ancho, sésiles, de borde liso, glabras, las del escapo floral lineares (2-4 cm de largo en la base de la inflorescencia) y generalmente secas al momento de la floración. Inflorescencias de 8-12 cm de largo, con 2-3 rayos de 5-7 cm de largo, bifurcados, con pedicelos de $2-2,5 \mathrm{~cm}$ de largo, que llevan 1-2 flores cada uno, de color violáceo a rosado intenso a pálido. Tépalos externos de 2,5-3,5 cm de largo, por 0,8-1,3 $\mathrm{cm}$ de ancho, obovados, con mucrón inflexo oscuro o verdoso, de 1-3 mm de largo, con rayas gruesas, cortas, paralelas entre sí, color atropurpúreo, en la mitad superior y oblicuas en el borde que es liso a levemente denticulado; a veces no presentan las rayas. Tépalos internos un poco más largos que los externos, de 3,2-3,7 cm de largo, más angostos, de forma oblanceolado-espatulada, con mucrón inflexo oscuro o verdoso, con rayas atropurpúreas en toda su extensión, que se van acortando hacia la base, los superiores rara vez con una débil franja o tonalidad amarilla en la parte media superior y en la base, el inferior de la forma y tamaño de los superiores, a veces levemente más ancho, y con la misma ornamentación, rara vez sin ella. Estambres de anteras violáceas.

Cápsula globosa, de 1,5-1,8 cm de largo, por $1,5 \mathrm{~cm}$ de ancho, color café claro, con 6 nervios oscuros, coronada por el estilo de $5 \mathrm{~mm}$ de largo. Semillas numerosas, redondeadas, de color café.

Florece a fines de diciembre y enero.

Planta de colorido variable, en pequeños grupos a orillas del camino, a veces bajo plantación de Eucalyptus, donde pueden conservar las hojas, o en lugares abiertos y asoleados (hojas secas en floración), en suelos compactos con piedras redondeadas, junto a quillay, litre, espino, boldo y gramíneas ya secas durante la floración de la planta (Fig. 2). Crece en el valle del río Achibueno, en la unión de este río con el Ancoa y en el curso inferior de este último, en su unión con el río Loncomilla,en el sector de La Balsa, en la Región del Maule, provincia de Linares, entre 240 y 390 m s.n.m. (Fig. 3.).

El autor en el protólogo indica: "especie que se puede confundir con A. revoluta R. et P. y A. timida Rav., de la primera se separa por el perigonio rosado (no pálido violáceo) y tépalos menos recurvados y de la segunda por las hojas tallinas no rosuladas y tépalos internos claramente rosados". En cuanto a A. timida (Ravenna, 1988) dice de "perigonio albo o rosa diluido", y está descrita de ejemplar cultivado, proveniente de la localidad monte Roble Alto, Polpaico. En dicha localidad, según García 2010 (Flora de Chicauma), crece A. garaventae, descrita antes por Bayer (1987), y que hemos observado, a veces presenta flores albas o rosado pálido, como Ravenna indica para su especie (Fig. 4).

Por la ornamentación es semejante a A. garaventae Ehr. Bayer, subsp. garaventae, pero aquella es planta más robusta, con tépalos más largos (3,5-5,5 mm de largo) (Fig. 3). La distribución de esta última es en localidades entre 1.200 y $2.000 \mathrm{~m}$ s.n.m. en el límite entre las regiones de Valparaíso y la Metropolitana y en la de O'Higgins (Fig. 4.) y existe un registro fotográfico para el portezuelo de la cuesta que une Chincolco con Alicahue (Región de Valparaíso) (Novoa 2007).

La subespecie aquí descrita se presenta $1,5^{\circ}$ de lat. más al sur que la subsp. garaventae y está presente en altitudes más bajas que la típica. Con este nuevo taxón se llega a 59 para Chile.

Para un mejor conocimiento de las subespecies se agrega una Tabla que indica las diferencias:

TABLA 1. Diferencias entre las subespecies. / Subspecies differences.

\begin{tabular}{lccc}
\hline \multicolumn{1}{c}{ Especie } & Largo tépalos & $\begin{array}{c}\text { Largo hojas base } \\
\text { inflorescencia }\end{array}$ & Altitud \\
\hline A garaventae subsp. garaventae & $3,5-5,5 \mathrm{~cm}$ & $8-9,5 \mathrm{~cm}$ & $1200-2000 \mathrm{~m}$ \\
A garaventae subsp. longaviensis & $2,5-3,5 \mathrm{~cm}$ & $2-4 \mathrm{~cm}$ & $240-390 \mathrm{~m}$ \\
\hline
\end{tabular}


En cuanto a su estado de conservación, por su distribución, estaría protegida en el Santuario de la Naturaleza Achibueno (creado 18 mayo 2015). En el resto de las localidades donde fue recolectada no tiene protección, ya que son predios privados destinados a diversos fines.

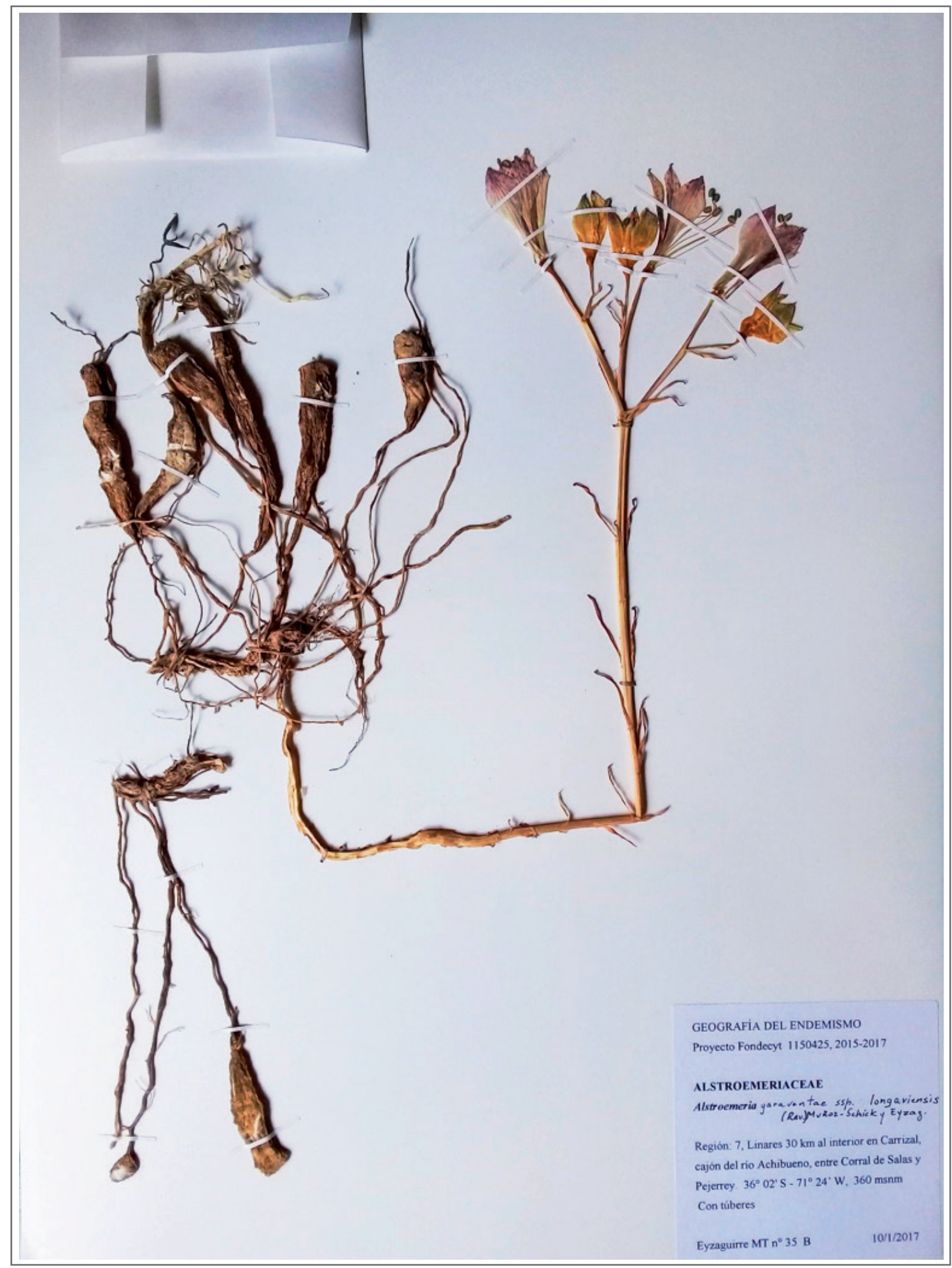

FIGURA 1. Alstroemeria garaventae subsp. longaviensis, detalle del sistema radicular (Eyzaguirre $35 \mathrm{~B}, \mathrm{SGO}$ ). / Alstroemeria garaventae subsp. longaviensis, radicular system details (Eyzaguirre 35 B, SGO). 

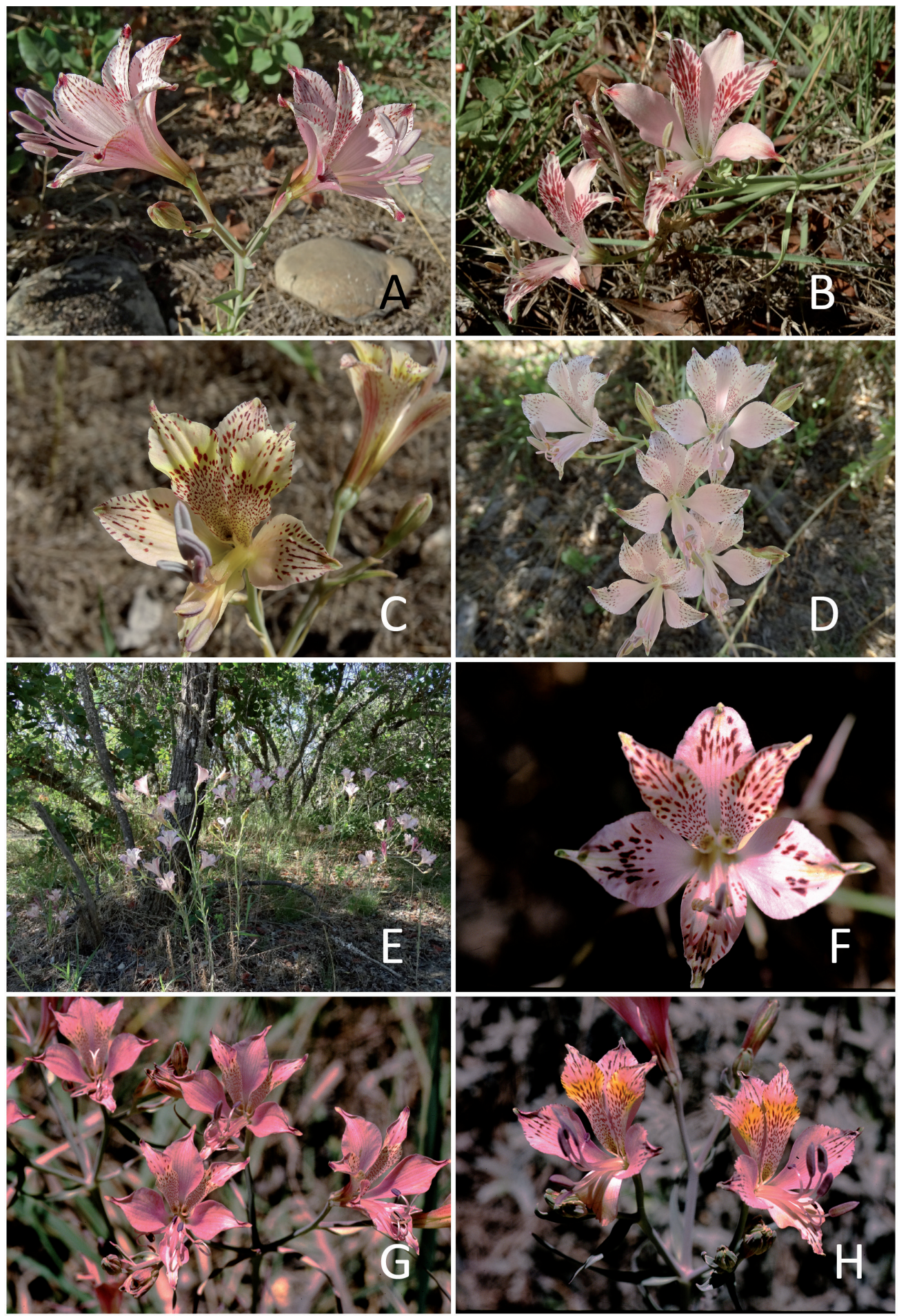

Figura 2. Alstroemeria garaventae subsp. longaviensis. A-D) detalle flores, Carrizal, cajón del río Achibueno, enero 2017. Fotos María Teresa Eyzaguirre; E) hábitat, Carrizal, cajón del río Achibueno, enero 2017. Foto María Teresa Eyzaguirre; F) detalle flores, Región del Maule, Linares, camino a embalse Ancoa, pasado puente Los Arcos, enero 2005. Foto Sergio Moreira; G-H) detalle flores, Ancoa, camping Los Copihues, enero 2016. Fotos Sergio Moreira. / Alstroemeria garaventae subsp. longaviensis. A-D) flower details, Carrizal, cajón del río Achibueno, january 2017. Photos María Teresa Eyzaguirre; E) habitat, Carrizal, cajón del río Achibueno, january 2017. Photo María Teresa Eyzaguirre; F) flower details, Región del Maule, Linares, road to embalse Ancoa, past puente Los Arcos, january 2005. Photo Sergio Moreira. G-H) flower details, Ancoa, camping Los Copihues, january 2016. Photos Sergio Moreira. 


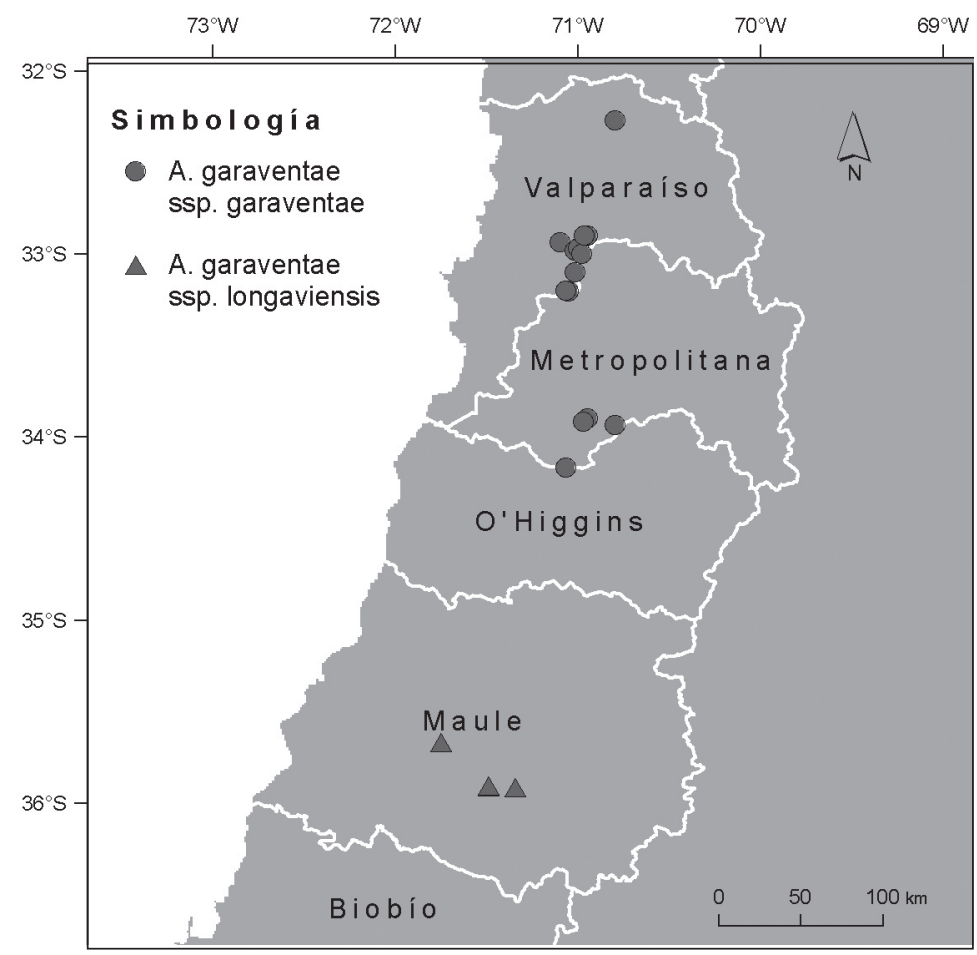

Figura 3. Mapa. Alstroemeria garaventae, subsp. Dibujado por V.D. Morales. / Map. Alstroemeria garaventae, subsp. Drew by V.D. Morales.
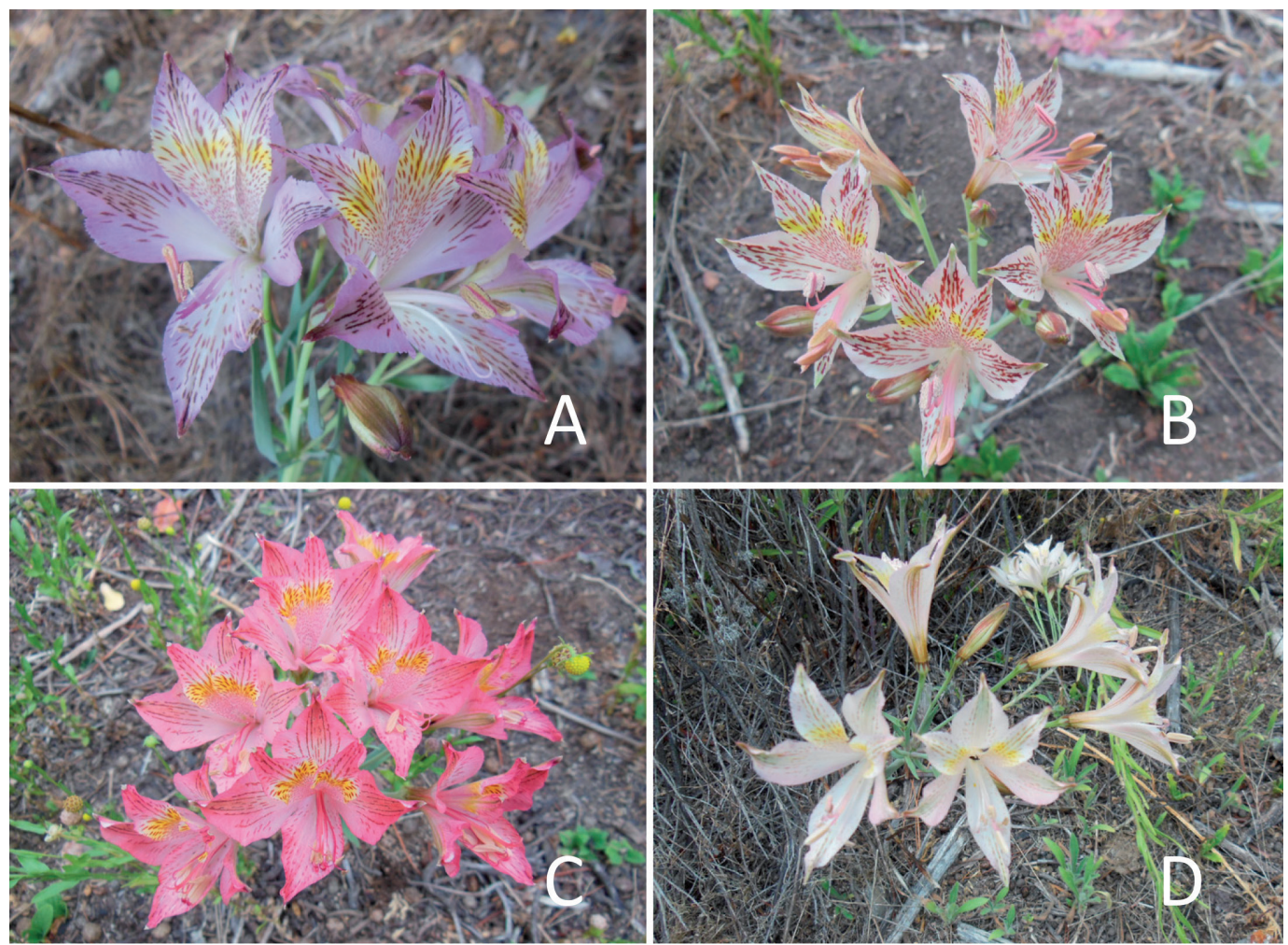

Figura 4. Alstroemeria garaventae subsp. garaventae. A-D) detalle flores, Región Metropolitana, Caleu, La Capilla, diciembre 2016. Fotos Mélica Muñoz. / Alstroemeria garaventae subsp. garaventae. A-D) flower details, Región Metropolitana, Caleu, La Capilla, dicember 2016. Photos Mélica Muñoz. 


\section{MATERIAL ESTUDIADO}

Región del Maule, Provincia Linares, Camino a Embalse Ancoa, camino a la izquierda, pasado puente Los Arcos, 243 m s.n.m., $35^{\circ}$ 54' 32,6" S - 71² 29' 24,6" W, Muñoz -Schick 4499, 5-I2005 (SGO 153734); ídem, 240 m s.n.m., 35 54' 30,2" S $71^{\circ} 29^{\prime} 9,2^{\prime \prime}$ W, Muñoz-Schick 4500 (SGO 153735); ídem Muñoz-Schick 4501 (SGO 153736); Camino desde Embalse Ancoa hacia poblado de Ancoa, km 13,7, $35^{\circ} 55^{\prime} 10,4^{\prime \prime} \mathrm{S}$ - 71' 21' 0,7" W, 362 m s.n.m., Muñoz-Schick 5629, 13-I2016 (SGO, CONC); Embalse Ancoa, camping, 389 m s.n.m., Arriagada s/n, 2-I-2011 (SGO 160722); Linares, precordillera, camino a Vado Azul, Arriagada s/n, 15-I-2012 (SGO 161365); Linares, El Peñasco, $22 \mathrm{~km}$ hacia la cordillera, $35^{\circ} 56^{\prime}-71^{\circ}$ 30', Arriagada 56, 57, 1-01-1996 (SGO 136823 y 136824); Linares $30 \mathrm{~km}$ al interior, en Carrizal, cajón del río Achibueno, entre Corral de Salas y Pejerrey, 360 m s.n.m., $36^{\circ} 2^{\prime} \mathrm{S}-71^{\circ}$ 24' W, Eyzaguirre 35, 35 A y 35 B, 10-I-2017 (SGO); Linares, Villa Alegre, sector Esperanza, fundo San Sebastián, $35^{\circ}$ 42'- 71 37', Arriagada s/n, 1-11-2011 (SGO 162556); Villa Alegre, al lado del rio Loncomilla, en el Sector La Balsa, $35^{\circ}$ $40^{\prime} \mathrm{S}-71^{\circ} 45^{\prime} \mathrm{W}$, Arriagada 29, 30-12-1995 (SGO 136795).

\section{AGRADECIMIENTOS}

A Jimena Arriagada, del Museo Nacional de Historia Natural, por recolectar también la planta en la provincia de Linares y a Gloria Rojas, jefa del Área Botánica, por la consulta de los ejemplares de SGO. A Eric y David Santos por monitorear la floración de la especie y por guiar en terreno a una de las autoras. A Sergio Moreira por acompañar a terreno a otra de las autoras y por las fotografías de la especie. A Vanezza Morales por el mapa de distribución. Proyecto Fondecyt 1180211.

\section{REFERENCIAS}

Baeza, C.M., Finot, V.L., Ruiz, E., Carrasco, P., Novoa, P., Rosas, M. 2016. Análisis citotaxonómico del complejo Alstroemeria diluta E. Bayer (Alstroemeriaceae) de Chile. Gayana Botánica 73(2): 220-225.

Bayer, E. 1987. Die Gattung Alstroemeria in Chile. Mitteilungen aus der Botanischen Staatssammlung München 24: 1362.

Finot, V., Baeza, C., Muñoz-Schick, M., Ruiz, E., Espejo, J., Alarcón, D., Carrasco, P., Novoa, P., Eyzaguirre, M.T. 2018. Guía de campo, Alstroemerias Chilenas. CORMA, $295 \mathrm{pp}$.

García, N. 2010. Caracterización de la flora vascular de Altos de Chicauma, Chile (33 S). Gayana Botánica 67(1): 65-112.

Muñoz-Schick, M., Moreira-Muñoz, A. 2003. Alstroemerias de Chile: diversidad, distribución y conservación. Taller La Era, Santiago. 139 pp.

Novoa, P. 2007. Nueva localidad y nuevo límite norte de distribución geográfica para Alstroemeria garaventae Ehr. Bayer (Alstroemeriaceae), una especie endémica para Chile. Chloris Chilensis 10(2). URL: http://www. chlorischile.cl.

Ruiz, E., Balboa, K., Negritto, M.A., Baeza, C.M., Fuentes, G., Briceño, V. 2010. Variabilidad genética y morfológica y estructuración poblacional en Alstroemeria hookeri subsp. hookeri (Alstroemeriaceae), endémica de Chile. Revista Chilena de Historia Natural 83: 605-616.

Ravenna, P. 1988. New or noteworthy species of Alstroemeria (Alstroemeriaceae). Phytologia 64(4): 281-288.

Received: 03.10.2018

Accepted: 15.04.2019 